\title{
Online Fault Detection Methods for Chillers Combining Extended Kalman Filter and Recursive One-class SVM
}

\author{
Ke Yan ${ }^{\mathrm{a}}$, Zhiwei Ji ${ }^{\mathrm{b}, *}$, Wen Shen ${ }^{\mathrm{c}}$ \\ ${ }^{a}$ College of Information Engineering, China Jiliang University, 258 Xueyuan Street, \\ Hangzhou, China, 310018 \\ ${ }^{b}$ School of Information and Electronic Engineering, Zhejiang Gongshang University, 18 \\ Xuezheng Road, Hangzhou, China, 310018 \\ ${ }^{c}$ Department of Informatics, University of California Irvine, Irvine, CA 92697
}

\section{Abstract}

Automatic, accurate and online fault detection of heating ventilation air conditioning (HVAC) subsystems, such as chillers, is highly demanded in building management system (BMS) to prevent energy waste and high maintenance cost. However, most fault detection techniques require rich faulty training data which is usually unavailable. In this study, a novel hybrid method is proposed to detect faults for chiller subsystems without any faulty training data available, i.e. by training the normal data only. A hybrid feature selection algorithm is applied to the chiller dataset collected by ASHRAE project 1043-RP to select the most significant feature variables. An online classification framework is introduced by combining an Extended Kalman Filter (EKF) model and a recursive one-class support vector machine (ROSVM). Experiment results show that the proposing algorithm detects typical chiller faults with high accuracy rates and requires less feature variables compared

\footnotetext{
${ }^{*}$ Corresponding author.

Email address: jzw18@hotmail.com (Zhiwei Ji)
}

Preprint submitted to Neurocomputing

July 18, 2016

(C) 2016. This manuscript version is made available under the Elsevier user license http://www.elsevier.com/open-access/userlicense/1.0/ 
to existing works.

Keywords: Chiller, Fault Detection, One-class Support Vector Machine, Extended Kalman Filter

\section{Introduction}

Under the current situation of global warming, the increasing energy portion consumed by heating, ventilation and air conditioning (HVAC) systems over the whole world urges us to develop efficient and effective monitoring system, such as the computer-aided HVAC fault detection and diagnosis (FDD) system. Compared to fault diagnosis, fault detection for HVAC system is more demanding, since it prevents the energy waste by detecting faults at early stages. In hot and humid regions, such as Abu Dhabi, the HVAC load represents nearly $70 \%$ of the total energy consumption according to a recent study by the local government agency [1]. Early detection of HVAC faults results in great energy, maintenance and financial savings.

Chiller is probably the most important and sophisticate component for a large-scale HVAC system, which includes an evaporator, a condenser and a compressor [2]. Chillers contribute the heat exchange load by providing chilled water to other HVAC subsystems, such as air handling units (AHUs), and then re-circulating the heated water to become chilled again. Without proper maintenance, a chiller may gradually lose the functionality in cooling re-circulated water, which results in increasing the supply chilled water temperature and completely ruining the HVAC cooling subsystem. Therefore, early fault detection is always demanded for chillers.

Data-driven fault detection techniques based on large-scale databases 
have been heavily studied and developed in recent years. Feature variables, such as the temperatures of the condenser water in/out, the water flow rates of the evaporator water in/out and valve positions, are recorded in constant time interval and stored in the database for a relatively long time period. Conventional fault detection methods take the historical data as training dataset and set threshold for each feature variable. Abnormal data varyings are identified as faults while the thresholds are exceeded. Existing datadriven fault detection approaches of chillers can be divided into two categories: offline and online [3]. Offline fault detection system utilizes the large amount of experimental data from laboratories and usually achieves high accuracy rates because of the richness of the training data [4, 5]. Online fault detection systems, to differentiate from offline fault detection systems, require to detect new fault types without training that particular faulty data samples. Moreover, the online fault detection system is required to detect faults soonest possible to avoid high maintenance costs.

In this study, we consider the situation that a normal operating chiller falls into faulty status gradually. First, an online fault detection algorithm is proposed, which recursively refines an one-class support vector machine (ROSVM). A time series dataset recorded by ASHRAE project 1043-RP [2] is used to demonstrate the effectiveness of the proposing algorithm. In the training phase, only normal data is utilized to build the support vectors in high dimension. The faulty data is identified as outliers in anomaly detection process. Second, the ROSVM approach is further improved by a hybrid method, where the data is first processed by an Extended Kalman Filter (EKF) before input into the ROSVM. The original dataset is converted into 
a set of parameter vectors before classified by ROSVM. Experimental results show that the proposing algorithm produces fault detection rates mostly higher than 80 percent, which are suitable for real-world application usage. The detailed contributions of our work are listed as follows:

- Hybrid feature selection algorithm. Feature selection is a preprocessing process of the original data. In this study, we introduce a hybrid feature selection algorithm that combines ReliefF and adaptive genetic algorithm (AGA) methods. The original sixty-four feature variables are reduced to six. In the real-world application system, only six sensors are required for the whole system to work, leading to great financial savings and convenient installations.

- Improved statistical model. The EKF is a statistical model which takes the original dataset as input and summarizes the data using functions. It is extremely helpful to filter out the noise samples and convert the original data samples into parameter vectors to stationarize the time series data. The data-processing by EKF is also an important step to potentially separate the normal and faulty data samples for the classifier. Although the EKF is already explored in the literature, the combination of EKF and ROSVM is treated as an improved version of our previous works $[6,7]$.

- Recursive one-class SVM. In contrast to existing works which employ the support vector data description (SVDD) method [8, 9], we implement a recursive one-class SVM to detect faults for chillers. The ROSVM refines the hyper-planes in high dimension by absorbing nor- 
mal tested data. Since the scenario of our work presumes that all faults become worse gradually to the severe levels, the normal data in the testing datasets are more meaningful because they are the most relevant normal data samples before the faults occur. By absorbing those normal data samples, the ROSVM produces higher classification accuracy rates for fault detection.

- Improved accuracy rates with less sensors required. Experimental results show that our method produces significantly higher classification accuracy rates with less sensor variables comparing with existing works. The high detection rates (mostly higher than 80 percent) significantly raise the possibility of implementing the online fault detection method in real-world building management systems (BMSs).

\section{Related Works}

Fault detection solutions for HVAC systems have been studied for decades. Recently, hybrid methods that combine statistical model and machine learning techniques become popular due to the stable detection and diagnosis rates. Li et al. [10] implemented a hybrid method to detect faults for air handling units (AHUs). Wang et al. [11] proposed a online fault detection and diagnosis (FDD) method which embeds a statistical model into a rule-based classifier. Bonvini et al. [12] studied Extended Kalman Filter and mixed the EKF with machine learning techniques. More recently, the residuals and parameter vectors are utilized in machine learning techniques for more accurate fault detection and classification. Yan et al. [6] converted data samples into parameter vectors and classified them using SVM to detect 
faults in chiller subsystems. The detection accuracy rates were significantly improved by employing the hybrid approaches. In the following year, the same team combined the auto-regressive with exogenous inputs (ARX) time series model with multi-class classifiers to detect faults for AHUs [7]. Experimental results showed that the hybrid technique combining the ARX model and SVM worked well for both chiller and AHU systems. Zhao et al. [8] first proposed to separate the original dataset into principal component (PC) space and residual (R) space using PCA. A hybrid method that combined the PC space and support vector data description (SVDD) was proposed to detect faults for chillers. Li et al. [9] improved Zhao's method by replacing the PC space parameters by $\mathrm{R}$ space parameters and named their hybrid method as PC-R-SVDD method.

Kalman Filter is a statistic model that transforms a series of observations with noises into a stationary time series measurements. The Kalman Filter and its extensions were used to detect fault for HVAC systems since the last century. In 1996, Yoshida et al. [13] started to detect HVAC faults using ARX model and EKF. Tudoroiu et al. [14] proposed an interactive multiple model augmented EKF to detect and diagnose faults for HVAC systems. In Bonvini et al.'s online FDD method [12], they add a back-smoothing component to EKF to increase the robustness of the FDD technique. Most recently, Mulumba et al. [15] developed a Kalman Filter based FDD technique for AHUs. The Kalman Filter is represented by an auto-regressive with exogenous inputs time series model with 17 parameters. The behavior of increasing or decreasing for any parameter gives hints for a rule-based system to determine the AHU working status. 
In 1995, Schiilkop et al. [16] introduced an one-class classifier which is represented by a hard-bounded hyper-sphere containing the training samples. In 1999, Tax and Duin [17, 18] introduced an extension of Schiilkop's method by replacing the hard-bounded hyper-sphere using a soft-bounded hyper-sphere and altered the method name as support vector domain/data description (SVDD) (Figure 1(a)). However, the spherical boundary of SVDD always suffers from non-spherical shape of the training dataset which produces too much empty space in the hyper-sphere. Schölkopf et al. [19] designed the one-class support vector machine (OSVM) which followed the idea of ordinary SVM. They searched for a set of hyper-planes which guarded the training dataset from the origin (Figure 1(b)). Utilizing the RBF kernel, the OSVM is proved equivalent to the SVDD classifier [20]. The OSVM is further improved in 2005 by introducing two hyper-planes to maximally gap the margin $[21,22]$.

\section{Online Fault Detection for Chillers}

In this work, we propose a recursive OSVM (ROSVM) based online fault detection system and a hybrid fault detection method which combines the ROSVM with EKF. In the ROSVM method, normal data samples are recursively inserted into the OSVM to simulate the normal operational condition for chillers. The OSVM forms support vectors in high dimension to bound normal data tightly. The faulty data samples are distinguished as outliers in anomaly detection process.

The ROSVM method is further improved by adding an EKF model before training and classification. We construct the EKF model based on normal 


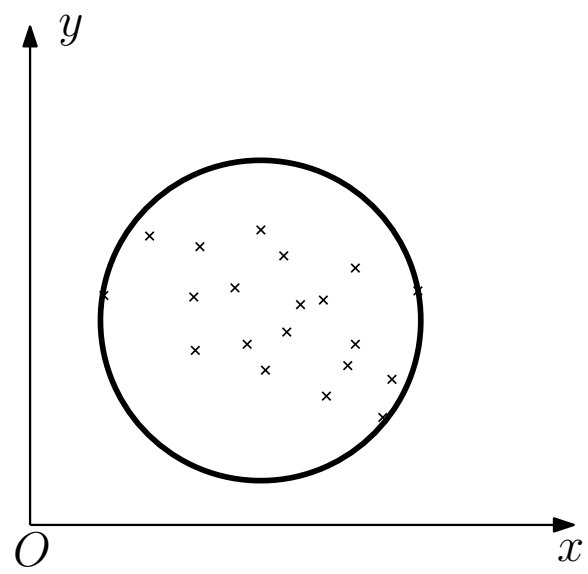

(a) SVDD

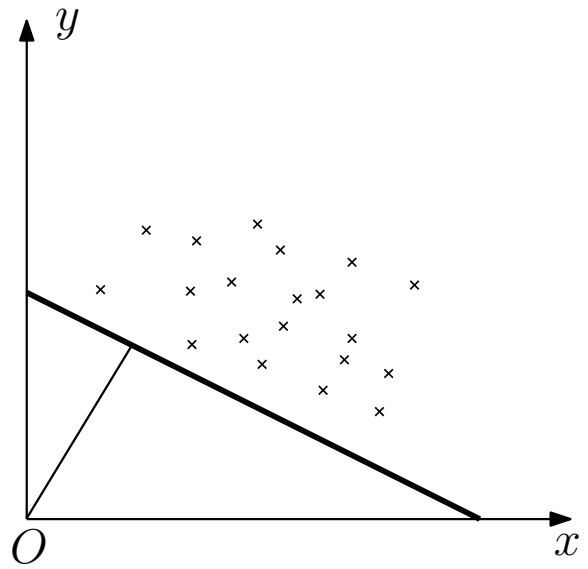

(b) OSVM

Figure 1: SVDD and OSVM approaches. In (a), a spherical hyper-plane is utilized to bounds the training sample set. The sphere shape restricts the SVDD usage while the training sample set is elongated. In (b), the hyper-plane separates the origin from training sample set. The OSVM is an alternative to the SVDD approach.

data only, and convert both normal and faulty data samples into parameter vectors. The experimental results show that the proposing EKF-ROSVM method produces significantly higher detection rates with less feature variables compared to existing works.

\subsection{An Overview of the EKF-ROSVM Approach}

An overview of the EKF-ROSVM approach is depicted in Figure 2. The chiller dataset from ASHRAE project 1043-RP [2] is used in this paper. The chiller dataset $X$ consists a set of normal conditional data $(x, 0)$ and a set of faulty data $\left(x^{*}, 1\right)$ both measured in a two-minute time interval, where 0 and 1 are status labels. We select six feature variables from the original set of sixty-four feature variables using a hybrid feature selection algorithm combining ReliefF and adaptive genetic algorithm (AGA). The EKF model 
is built based on half of the normal data samples. For each normal data sample $(x, 0)$, a parameter vector $p$ is generated by the EKF model. The parameter vector set $P$ that gathers all $p$ is used to train the ROSVM.

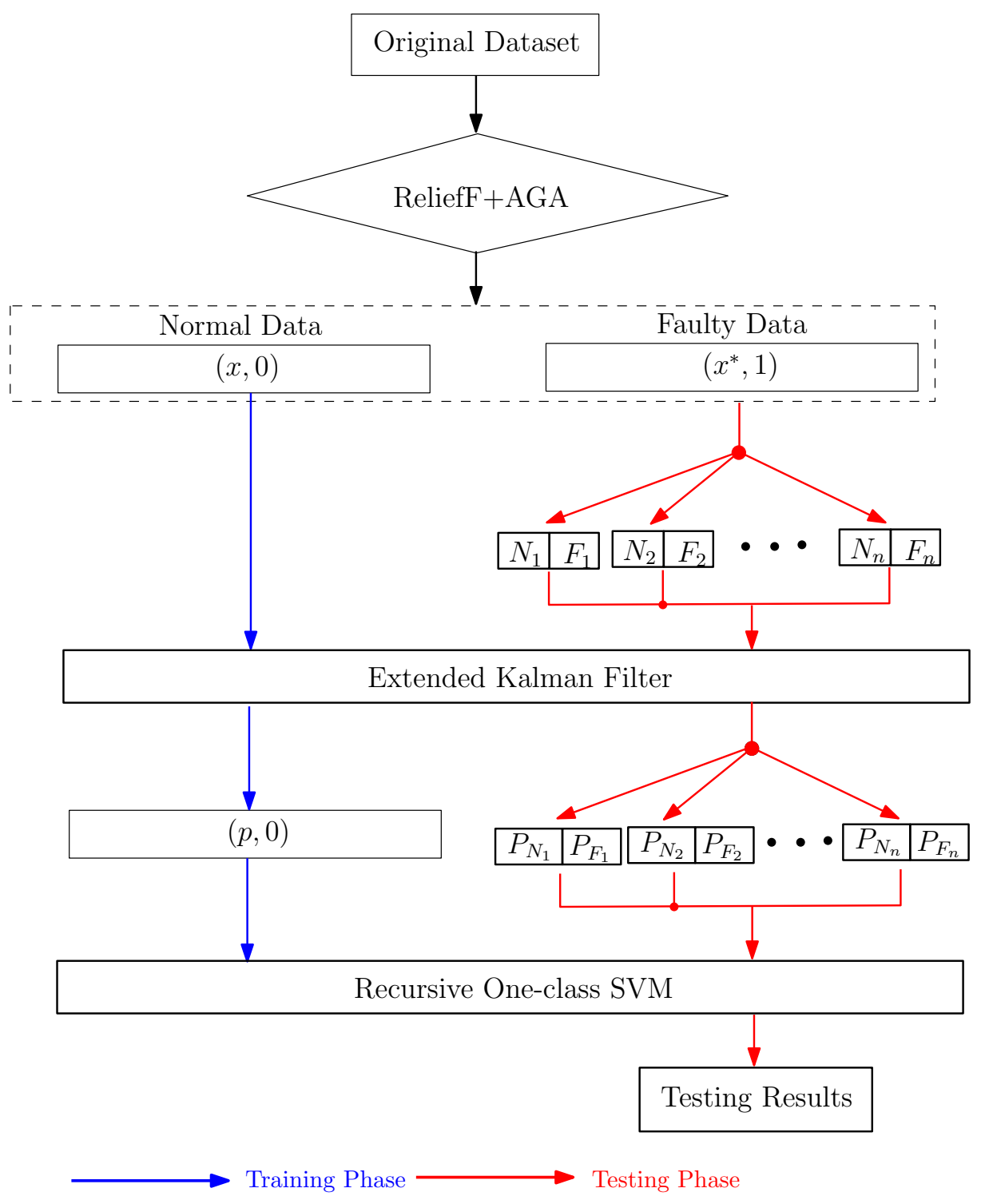

Figure 2: The flowchart of EKF-ROSVM approach.

In the testing phase, a series of testing datasets $N_{i} F_{i}$ are reformulated 
from the other half of the normal conditional dataset combining the faulty dataset, where i indicates different types of faults. Dataset pairs $N_{i} F_{i}$ are inserted into the EKF model to produce parameter vector sets $P_{N_{i}} P_{F_{i}}$. After removing the labels, each parameter vector $p \in P_{N_{i}} P_{F_{i}}$ is tested by the ROSVM. During the testing process, the ROSVM are refined by newly tested normal parameter vectors. Alarm messages are generated for faulty parameter vectors.

The EKF is a statistical model; and statistical models are proved to be powerful in modeling structural changes to detect faults for chillers and AHUs from our previous works [6, 7]. The statistical models are extremely advantageous while faults become worse gradually at a slow rate and data samples arrive in a sequence according to time. Compared to the original data samples, parameter vectors produced by the statistical methods are more distinguishable and thus more easily detectable by machine learning techniques, such as SVM.

\subsection{A Hybrid Feature Selection Method: ReliefF-AGA}

The original dataset collected by ASHRAE project 1043-RP consists of sixty-four feature variables that correspond to sixty-four remote sensors in a real-world fault detection application system. Hardware and financial restrictions prompt us to select the most important feature variables to reduce the number of sensors. In our previous works [6, 7], the ReliefF method [23, 24] is employed to select features with maximal information. In ReliefF, the information contained by a particular feature variable is measured by weight. We depicted the weights from ReliefF in Figure 3; and feature variables with weights greater than 1 are listed in Table 1. 


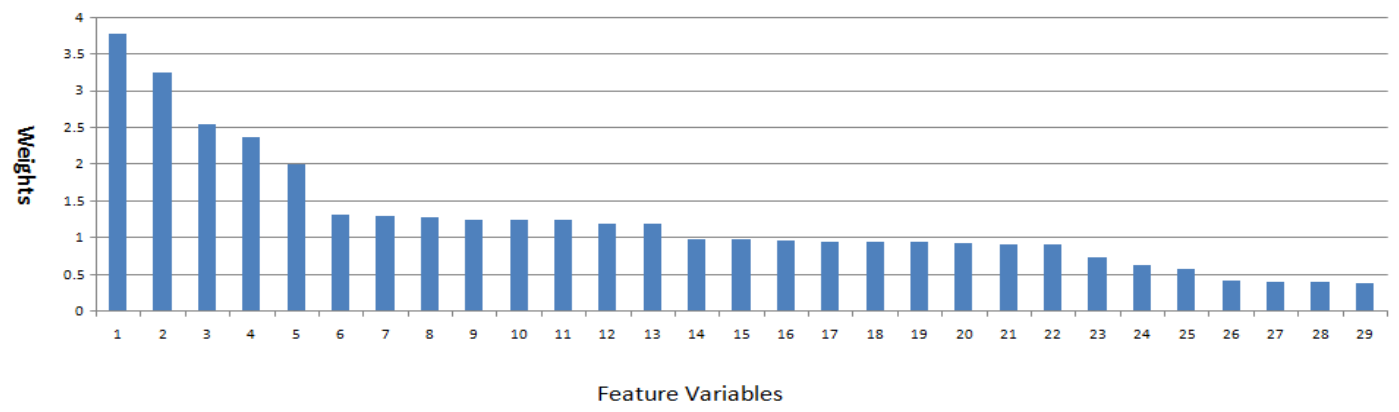

Figure 3: The results from the ReliefF feature selection algorithm.

Table 1: Top important feature variables from ReliefF feature selection method.

\begin{tabular}{ccc}
\hline Index & Feature & Description \\
\hline 1 & $k W$ & Instantaneous input power \\
2 & $T C O$ & Temperature of Condenser Water Out \\
3 & $T E I$ & Temperature of Evaporator Water In \\
4 & $T C I$ & Temperature of Condenser Water In \\
5 & $T E O$ & Temperature of Evaporator Water Out \\
6 & $P R E$ & Evaporator Pressure \\
7 & $P R C$ & Condensor Pressure \\
8 & $T R C_{\text {ssub }}$ & Subcooling \\
9 & $k W /$ ton & Chiller efficiency \\
10 & $T E A$ & Evaporator Approach Temperature \\
11 & EvapTons & Tons of cooling delivered by the evaporator coil \\
12 & $T C A$ & Condenser Approach Temperature \\
13 & Tsh_dis & Discharge Superheat \\
\hline
\end{tabular}

Although ReliefF provides the feature variables with the maximal information from the dataset. It ignores the co-relationship in-between the feature variables. In this study, the top thirteen most important feature variables (Table 1) are further reduced to six in Table 2 by considering minimizing the co-relations between feature variables. A hybrid feature selection algorithm 
which combines the ReliefF and adaptive genetic algorithm is proposed to achieve the target.

In 2011, Han et al. [5] first used the genetic algorithm (GA) as a feature selection algorithm to solve a fault detection problem for chillers. The GA is demonstrated to be an effective method reducing the number of feature variables from the original dataset. There are two major operations in GA, namely crossover and mutation. The features selected by GA depends on two user-defined probabilities, namely crossover probability $P_{c}$ and mutation probability $P_{m}$. In conventional GA, both $P_{c}$ and $P_{m}$ are set according to user expertise.

In this study, an adaptive GA (AGA) is employed and used similar to the GA method in [5]. The only difference is that we allow the two probabilities $P_{c}$ and $P_{m}$ to adjust themselves during the searching process according to the Equation 1 and 2:

$$
\begin{gathered}
P_{c}=\left\{\begin{array}{l}
k_{1} \cdot \frac{\left(f_{\max }-f^{\prime}\right)}{\left(f_{\max }-f_{\text {avg }}\right)}, \text { if } f^{\prime} \geq f_{\text {avg }}, \\
k_{2}, \text { if } f^{\prime}<f_{\text {avg }}
\end{array},\right. \\
P_{m}=\left\{\begin{array}{l}
k_{3} \cdot \frac{\left(f_{\max }-f\right)}{\left(f_{\max }-f_{\text {avg }}\right)}, \text { if } f \geq f_{\text {avg }}, \\
k_{4}, \text { if } f<f_{\text {avg }}
\end{array},\right.
\end{gathered}
$$

where $f_{\text {max }}, f_{\text {avg }}, f$ and $f^{\prime}$ represent the maximal fitness, average fitness, bigger fitness of the parents in mutation operation and bigger fitness of the parents in crossover operation respectively. Variables $k_{1}, k_{2}, k_{3}$ and $k_{4}$ are four control variables ranging from 0 to 1 . The AGA follows Algorithm 1 
$[25,26]$. Variables in Table 2 are outputs from the AGA and also inputs for the EKF.

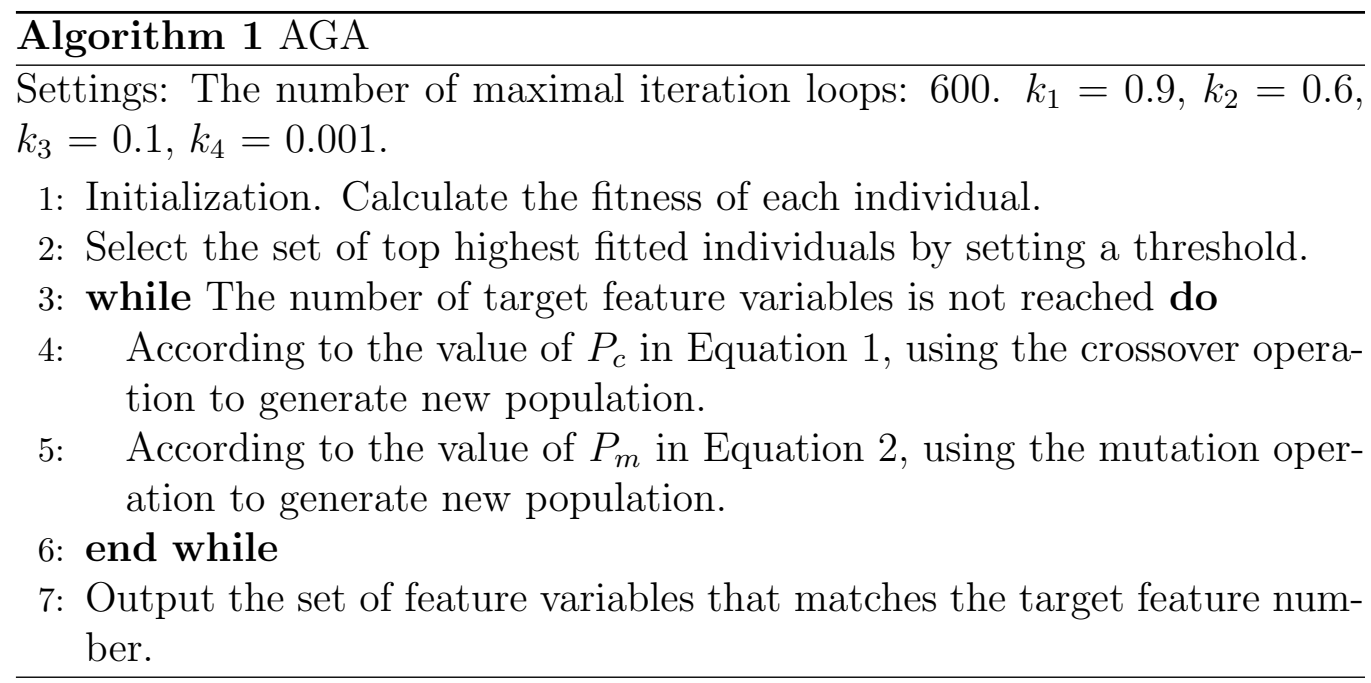

Table 2: Input variables for statistical models.

\begin{tabular}{ccc}
\hline Index & Feature & Description \\
\hline 1 & $k W$ & Instantaneous input power \\
2 & $T C O$ & Condenser Water Temperature Out \\
3 & $T E I$ & Evaporator Water Temperature In \\
4 & $T C I$ & Condenser Water Temperature In \\
5 & $T E A$ & Evaporator Approach Temperature \\
11 & EvapTons & Tons of cooling delivered by the evaporator \\
& & coil \\
\hline
\end{tabular}

\subsection{Extended Kalman Filter Model}

The EKF model is a statistical model providing stationarized parameter vectors that statistically conclude the original dataset. The hybrid method 
that combines the statistical models with SVM is proved to be useful in distinguishing faulty data from normal data in our previous works $[6,7]$. In this study, the EKF model is developed based on the work in [15], which consists of two steps: pre-whitening and model formulation.

Pre-whitening is a generalized form of feature normalization. It reduces the co-relations between feature variables by considering lag values. There are reports indicating that the pre-whitening is more suitable for non-Gaussian distribution [27]; and the data collected from ASHRAE 1043-RP is shown to be non-Gaussian [8]. After pre-whitening, the system becomes stationary while residuals are represented by fitted auto-regressive integrated movingaverage (ARIMA) models. The pre-whitening process can be described in the following two equations:

$$
\begin{aligned}
X(k) & =a \cdot X(k-1)+b \cdot U(k)+W(k), \\
Z(k) & =h \cdot X(k)+V(k),
\end{aligned}
$$

where $X(k)$ stands for $k W ; Z$ is the measured value of $X ; X(k-1)$ is the actual $X$ value in the previous time frame; $W(k), V(k)$ are both ARIMA models. The EKF predicts the object states by combining noisy measurements and can be applied to many linear or non-linear dynamic systems. It has recursive manner and refines itself by absorbing newly inserted data samples.

In this study, the maximum lag is selected as 2 in the pre-whitening step. The EKF model is implemented using Matlab toolboxes [28, 29] and 
formulated as an ARX model:

$$
\begin{array}{r}
\mathrm{kW}=\alpha_{1}+a_{t}+\alpha_{2} \mathrm{~kW}_{t-1}+\alpha_{3} \mathrm{~kW}_{t-2}+\alpha_{4} \mathrm{TCO}_{t}+\alpha_{5} \mathrm{TCO}_{t-1}+\alpha_{6} \mathrm{TCO}_{t-2} \\
+\alpha_{7} \mathrm{TEI}_{t}+a_{t}+\alpha_{8} \mathrm{TEI}_{t-1}+\alpha_{9} \mathrm{TEI}_{t-2}+\alpha_{10} \mathrm{TCI}_{t}+\alpha_{11} \mathrm{TCI}_{t-1}+\alpha_{12} \mathrm{TCI}_{t-2} \\
+\alpha_{13} \mathrm{TEA}_{t}+a_{t}+\alpha_{14} \mathrm{TEA}_{t-1}+\alpha_{15} \mathrm{TEA}_{t-2} \\
+\alpha_{16} \text { EvapTons }_{t}+\alpha_{17} \text { EvapTons }_{t-1}+\alpha_{18} \text { EvapTons }_{t-2} .
\end{array}
$$

After being processed by the EKF model, each data sample is converted into a vector of 18 parameters $\left(\alpha_{1}, \alpha_{2}, \ldots, \alpha_{18}\right)$.

The advantages of taking a data-processing by EKF include: filtering out the noise samples, stationarizing the time series data and making the data samples more distinguishable for the ROSVM. The limitation of the EKF is that it requires a transition time period for the whole system to reach stationary status. In this study, we simply eliminate the data samples and parameter vectors in the transition period to avoid the side-effects.

\subsection{Recursive One-class SVM}

\subsubsection{One-class $S V M$}

The one-class SVM (OSVM) takes substantial large amount of normal data, forms a single class and separates the rest of the feature space from this class by hyper-planes [19]. The separation is estimated by a function $f$ which is positive on training set and negative on the complement.

Suppose the training data set contains $x_{1}, \ldots, x_{l} \in X$ and $i, j \in[1, l]$. The separation function $f(x)$ maximizes the distance from the origin to the 
hyper-plane separating the training set in feature space $F$. The final goal is achieved by two steps. First, a quadratic equation is solved:

$$
\begin{gathered}
\min _{w, \xi_{i}, \rho} \frac{1}{2}\|w\|^{2}+\frac{1}{\nu n} \sum_{i=1}^{n} \xi_{i}-\rho, \\
\text { subject to : } \\
\left(w \cdot \phi\left(x_{i}\right)\right) \geq \rho-\xi_{i}, \quad \text { for all } i=1, \ldots, n, \\
\xi_{i} \geq 0, \quad \text { for all } i=1, \ldots, n,
\end{gathered}
$$

to determines the values of $w, \rho$ and $\nu$. Variables $w$ and $\rho$ create a hyperplane that maximizes the distance from the origin in $F$. The variable $\nu$ provides the upper bound on the fraction of outliers and the lower bound on the number of training samples. The function $f$ can be written as:

$$
f(x)=\operatorname{sgn}\left(\left(w \cdot \phi\left(x_{i}\right)\right)-\rho\right)=\operatorname{sgn}\left(\sum_{i=1}^{n} \alpha_{i} K\left(x, x_{i}\right)-\rho\right),
$$

which returns +1 in a tight region capturing most of the training data samples, and -1 for elsewhere in $F$.

\subsubsection{A Semi-Supervised Learning Method: ROSVM}

We alter the SVDD classifier used in $[8,9]$ by a semi-supervised learning method called recursive one-class SVM (ROSVM). The ROSVM method receives data samples in a constant time interval (in the case of ASHRAE reports $1043-\mathrm{RP}$ is two minutes). The starting data samples are presumed to be normal data. The OSVM takes the normal dataset as training dataset and do anomaly detection for faulty data. The online fault detection system is required to work under the condition of receiving both normal and faulty 
data samples, absorbing normal samples to refine the support vectors in high dimension and throwing alarm messages for faulty samples (Figure 4).

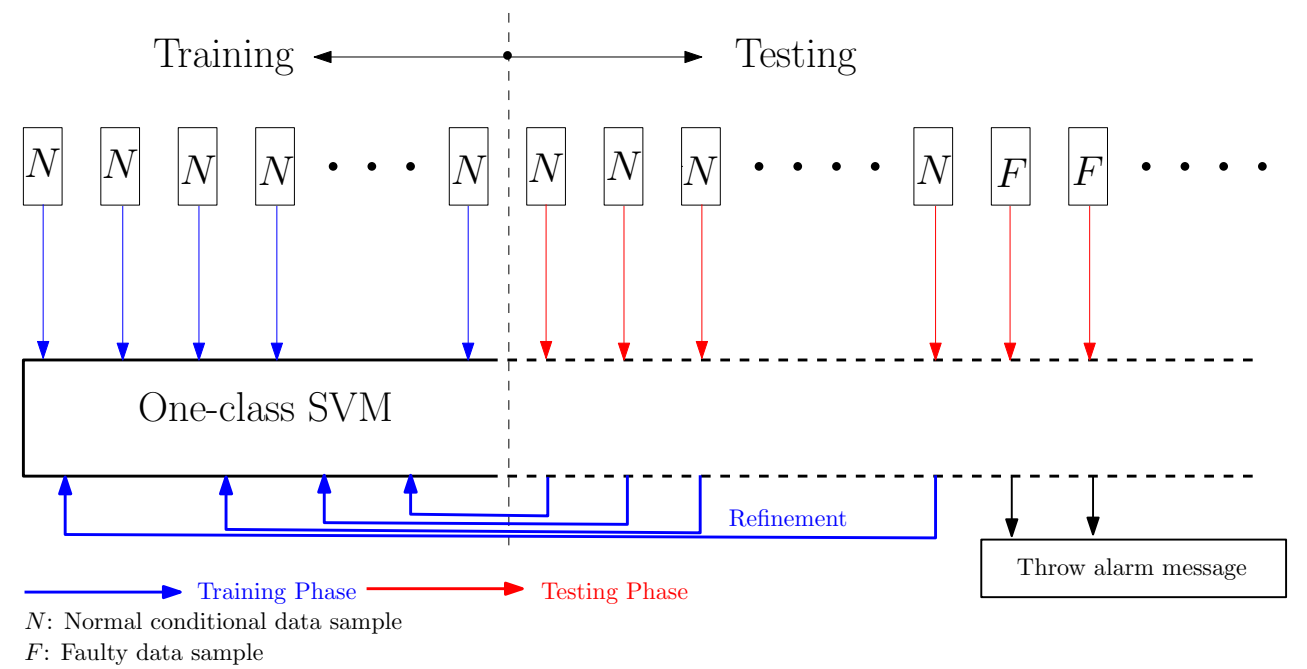

Figure 4: The working scenario for ROSVM.

We also show the working scenario of EKF-ROSVM (Figure 5). The EKF model, which is again built based on normal data, converts every data sample (including faulty data samples) into a vector of parameters. The EKF model is a dynamic statistical model which usually requires a certain time period to stationarize the time series dataset starting from the occurrence of faults. In this study, we assume that the stationarization process takes less than ten minutes which is five time steps. The data samples in this time period is neglected and used in neither the training nor the testing process.

\section{Experimental Results}

We divide the normal dataset in ASHRAE project 1043-RP evenly into two subsets. The first subset is the training dataset, which contains half 


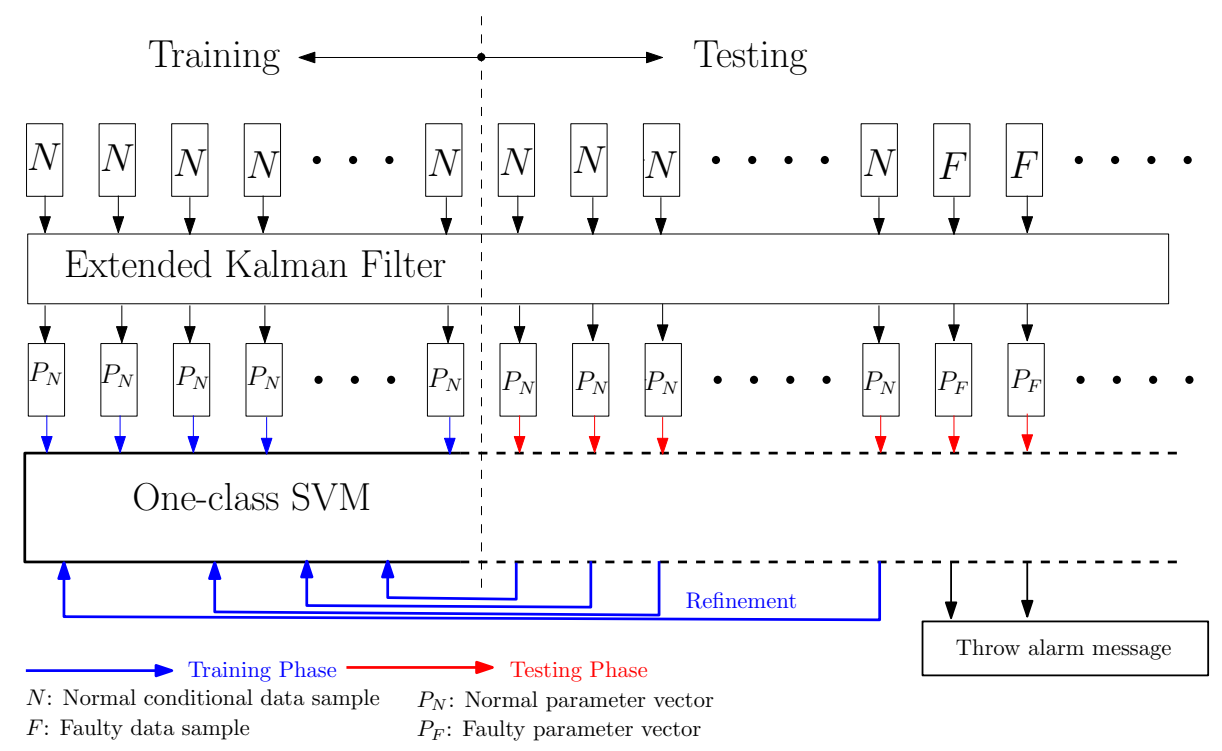

Figure 5: The working scenario for EKF-ROSVM.

of the normal data samples. The second subset is further divided into 20 subsets, each of which contains a normal dataset followed by a faulty dataset. Each faulty dataset corresponds to a specific fault type in a particular severe level in Table 3 [2]. The ROSVM is implemented by LibSVM [30] with a confidence level of $95 \%$ (the allowed true negative rate is $5 \%$ ).

Table 3: The 20 fault types inherited from ASHRAE project report 1043-RP [2], where SL represents severe level.

\begin{tabular}{cccccc}
\hline Faults & Description & SL1 & SL2 & SL3 & SL4 \\
\hline F1 & reduced condenser water flow & $-10 \%$ & $-20 \%$ & $-30 \%$ & $-40 \%$ \\
F2 & reduced evaporator water flow & $-10 \%$ & $-20 \%$ & $-30 \%$ & $-40 \%$ \\
F3 & refrigerant leak & $-10 \%$ & $-20 \%$ & $-30 \%$ & $-40 \%$ \\
F4 & condenser fouling & $-12 \%$ & $-20 \%$ & $-30 \%$ & $-45 \%$ \\
F5 & non-condensables in refrigerant & $-10 \%$ & $-20 \%$ & $-30 \%$ & $-40 \%$ \\
\hline
\end{tabular}

Two approaches are designed to detect the faults for chillers: 
- Approach 1 (ROSVM). The ROSVM is directly applied to the ASHRAE project 1043-RP dataset;

- Approach 2 (EKF-ROSVM). An EKF model is constructed to convert each data sample to a vector of 18 parameters. The ROSVM is used to train and detect faults using parameter vectors.

The EKF is a statistical model which requires a short time period to reach the stationary state when each fault occurs. In this study, we assume that the time period of a stationarization process is always shorter than 10 min (5 time steps). As a result, we always remove five data samples in the transition time period. Assumptions are made that the final classification accuracy is not affected much by removing the five transition samples.

In the experiment, thirteen feature variables in Table 1 are used for the ROSVM approach, which supposingly require thirteen remote sensors in realworld application systems. The EKF-ROSVM approach utilizes six feature variables which correspond to six remote sensors. Experimental results show that the EKF-ROSVM approach significantly improves the chiller fault detection rates (Tables 4 and 5).

Table 4: The fault detection accuracy rates with different levels and types of faults for ROSVM approach.

\begin{tabular}{ccccccc}
\hline $\begin{array}{c}\text { Accuracy } \\
\text { (\%) }\end{array}$ & $\begin{array}{c}\text { All } \\
\text { faults }\end{array}$ & F1 & F2 & F3 & F4 & F5 \\
\hline level 1 & 68.44 & 77.29 & 66.53 & 63.59 & 58.76 & 74.68 \\
level 2 & 72.78 & 82.65 & 68.09 & 70.77 & 66.83 & 82.42 \\
level 3 & 83.32 & 86.83 & 76.46 & 79.95 & 78.90 & 87.90 \\
level 4 & 90.06 & 91.06 & 82.83 & 84.96 & 86.15 & 94.74 \\
\hline
\end{tabular}


Table 5: The fault detection accuracy with different levels and types of faults for EKFROSVM approach.

\begin{tabular}{ccccccc}
\hline $\begin{array}{c}\text { Accuracy } \\
\text { (\%) }\end{array}$ & $\begin{array}{c}\text { All } \\
\text { faults }\end{array}$ & F1 & F2 & F3 & F4 & F5 \\
\hline level 1 & 85.24 & 91.50 & 87.26 & 85.62 & 62.07 & 80.37 \\
level 2 & 88.42 & 87.58 & 92.36 & 90.85 & 79.31 & 82.62 \\
level 3 & 89.11 & 95.42 & 92.86 & 93.46 & 81.87 & 88.12 \\
level 4 & 93.59 & 94.12 & 92.99 & 95.42 & 91.25 & 95.47 \\
\hline
\end{tabular}

We compare the two approaches with the PCA-PC-SVDD approach [8] and the PCA-R-SVDD approach [9] for severe levels 1 and 2 (Figures 6 and 7). It is noted that the results from PCA-PC-SVDD and PCA-R-SVDD are obtained from utilizing eight feature variables from the original ASHRAE 1043-RP dataset. The ROSVM approach utilizes thirteen feature variables from the same dataset, whereas the EKF-ROSVM method only uses six. The EKF-ROSVM method provides detection accuracy improvement over other methods using less feature variables and sensors. Moreover, some variables in Table 1 can be difficult to measure using sensors.

\section{Conclusion and Discussion}

This work first proposes a ROSVM algorithm to detect typical chiller faults. The most important contribution of this work is that the faulty data is not required in the training phase. In the experiment, we order the data samples in a way that the faults become worse gradually after a relatively long time period of normal operations. The experimental results are therefore close to the real-world cases since the faulty data is only used in the testing phase. The top thirteen most important feature variables are 


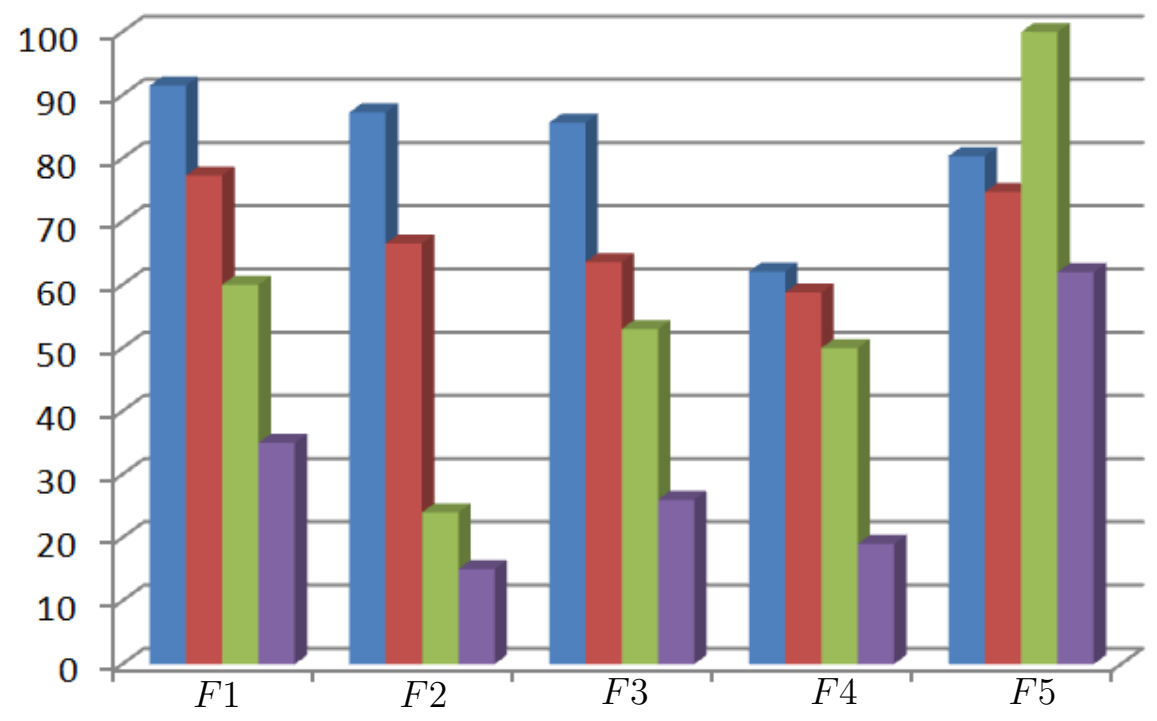

EKF-ROSVM

ROSVM

- PCA-R-SVDD

PCA-PC-SVDD

Figure 6: The accuracy rates comparison between EKF-ROSVM, ROSVM, PCA-PCSVDD and PCA-R-SVDD for severe level 1.

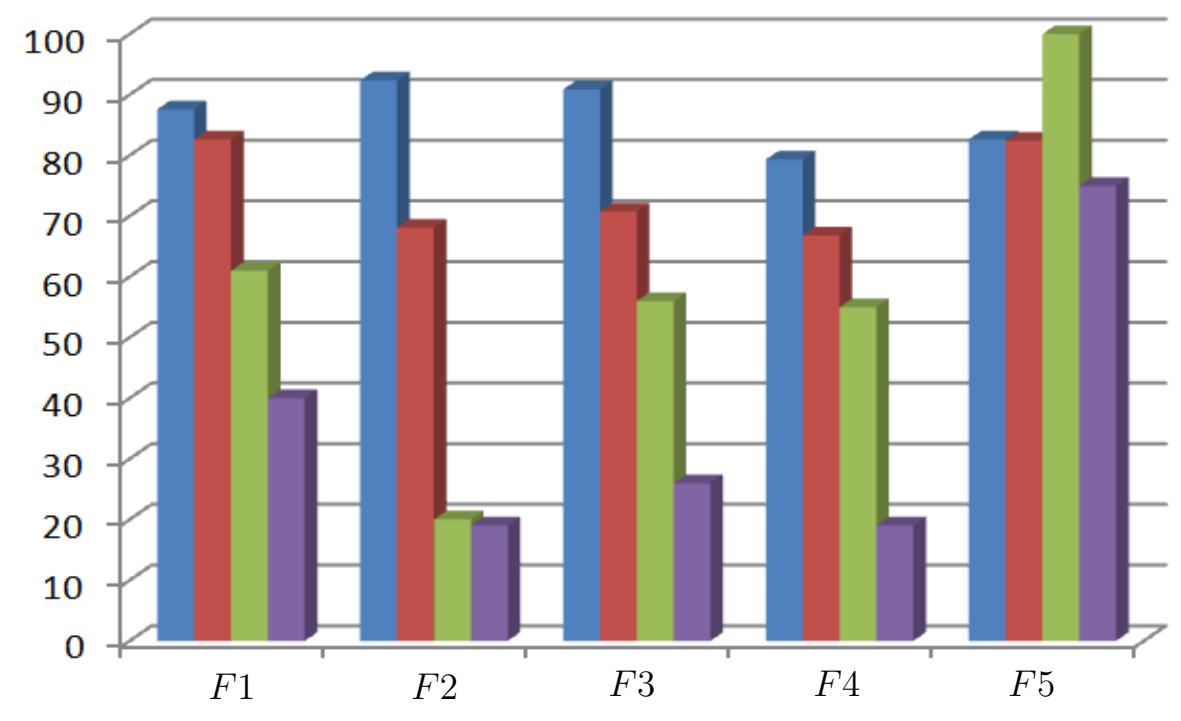

EKF-ROSVM

- ROSVM

- PCA-R-SVDD

- PCA-PC-SVDL

Figure 7: The accuracy rates comparison between EKF-ROSVM, ROSVM, PCA-PCSVDD and PCA-R-SVDD for severe level 2. 
selected by a hybrid feature selection algorithm and utilized in the whole process of ROSVM. In the testing phase, recognized normal data samples are recursively inserted into the one-class SVM to improve the detection accuracy rates. A set of data collected from a laboratory 90-ton centrifugal chiller of ASHRAE project number 1043-RP is utilized to demonstrate the effectiveness of the proposing method.

The ROSVM method is further improved to a hybrid EKF-ROSVM method by pre-processing the data using a statistical model before inserting the data into ROSVM. In this study, the statistical model is selected to be an EKF model. In fault detection problems for chillers, data are usually received sequentially according to time and fault become worse gradually. The EKF model provides more significant parameter vectors statistically concluding the original dataset. By using EKF-ROSVM, we achieve detection rates mostly in between of $80 \%$ to $95 \%$ (except F4 severe level 1 and 2) for typical chiller faults using only 6 sensors. With a reduced number of features, the deployment on a multitude of operating plants requires only the selected sensors for both training and testing, which results in significant savings.

The proposing method makes two assumptions for the whole fault detection system to work. First, we assume that there is always a relatively long time period of normal data available before any fault occurs; and the faults always become worse gradually. We claim that the first assumption is valid even in real-world scenarios. Second, we assume that the transition data samples from normal to faulty do not affect the overall detection accuracy much, since we neglect the data samples in that period. Future works will 
be conducted to deal with the data samples during the transition period.

\section{Conflict of Interests}

All authors declare that there is no conflict of interest regarding the publication of this manuscript.

\section{Acknowledgement}

Authors would like to send their special thanks to Dr Afshin Afshari from Masdar Institute of Science and Technology, Abu Dhabi, UAE, for his con-

tinuous support and constructive advices to this project. We are also grateful to the authors of ASHRAE project 1043-RP for providing the chiller dataset.

\section{References}

[1] A. D. Municipality, Energy Consumption Management Program in Existing Buildings, personal communication, 31 October 2011.

[2] M. C. Comstock, J. E. Braun, R. Bernhard, Experimental data from fault detection and diagnostic studies on a centrifugal chiller, Purdue University, 1999.

[3] J. Cui, S. Wang, A model-based online fault detection and diagnosis strategy for centrifugal chiller systems, International Journal of Thermal Sciences 44 (10) (2005) 986-999.

[4] H. Han, B. Gu, Y. Hong, J. Kang, Automated FDD of multiplesimultaneous faults (MSF) and the application to building chillers, Energy and Buildings 43 (9) (2011) 2524-2532. 
[5] H. Han, B. Gu, J. Kang, Z. Li, Study on a hybrid SVM model for chiller FDD applications, Applied Thermal Engineering 31 (4) (2011) 582-592.

[6] K. Yan, W. Shen, T. Mulumba, A. Afshari, ARX model based fault detection and diagnosis for chillers using support vector machines, Energy and Buildings 81 (2014) 287-295.

[7] T. Mulumba, A. Afshari, K. Yan, W. Shen, L. K. Norford, Robust model-based fault diagnosis for air handling units, Energy and Buildings 86 (2015) 698-707.

[8] Y. Zhao, S. Wang, F. Xiao, Pattern recognition-based chillers fault detection method using Support Vector Data Description (SVDD), Applied Energy 112 (2013) 1041-1048.

[9] G. Li, Y. Hu, H. Chen, L. Shen, H. Li, M. Hu, J. Liu, K. Sun, An improved fault detection method for incipient centrifugal chiller faults using the PCA-R-SVDD algorithm, Energy and Buildings 116 (2016) 104-113.

[10] S. Li, J. Wen, Application of pattern matching method for detecting faults in air handling unit system, Automation in Construction 43 (2014) $49-58$.

[11] H. Wang, Y. Chen, C. W. Chan, J. Qin, J. Wang, Online model-based fault detection and diagnosis strategy for VAV air handling units, Energy and Buildings 55 (2012) 252-263.

[12] M. Bonvini, M. D. Sohn, J. Granderson, M. Wetter, M. A. Piette, Robust on-line fault detection diagnosis for HVAC components based on 
nonlinear state estimation techniques, Applied Energy 124 (2014) 156166.

[13] H. Yoshida, T. Iwami, H. Yuzawa, M. Suzuki, Typical faults of air conditioning systems and fault detection by ARX model and extended Kalman filter, Tech. Rep., American Society of Heating, Refrigerating and Air-Conditioning Engineers, Inc., Atlanta, GA (United States), 1996.

[14] N. Tudoroiu, M. Zaheeruddin, E.-R. Tudoroiu, V. Jeflea, Fault detection and diagnosis (FDD) in heating ventilation air conditioning systems (HVAC) using an interactive multiple model augmented unscented Kalman filter (IMMAUKF), in: 2008 Conference on Human System Interactions, IEEE, 334-339, 2008.

[15] T. Mulumba, A. Afshari, L. Friedrich, Kalman filter-based Fault Detection and Diagnosis for Air Handling Units, in: Proceedings of 15th International Refrigeration and Air Conditioning Conference, 2014.

[16] P. Schiilkop, C. Burgest, V. Vapnik, Extracting support data for a given task, in: Proceedings of the 1st International Conference on Knowledge Discovery \& Data Mining, 252-257, 1995.

[17] D. M. Tax, R. P. Duin, Support vector domain description, Pattern recognition letters 20 (11) (1999) 1191-1199.

[18] D. M. Tax, R. P. Duin, Support vector data description, Machine learning 54 (1) (2004) 45-66. 
[19] B. Schölkopf, R. C. Williamson, A. J. Smola, J. Shawe-Taylor, J. C. Platt, et al., Support Vector Method for Novelty Detection., in: NIPS, vol. 12, Citeseer, 582-588, 1999.

[20] B. Schölkopf, J. C. Platt, J. Shawe-Taylor, A. J. Smola, R. C. Williamson, Estimating the support of a high-dimensional distribution, Neural computation 13 (7) (2001) 1443-1471.

[21] J. Giesen, S. Spalinger, B. Schölkopf, Kernel methods for implicit surface modeling, in: Advances in neural information processing systems, 11931200, 2004.

[22] Q. Tao, G.-w. Wu, J. Wang, A new maximum margin algorithm for oneclass problems and its boosting implementation, Pattern Recognition 38 (7) (2005) 1071-1077.

[23] M. Robnik-Šikonja, I. Kononenko, Theoretical and empirical analysis of ReliefF and RReliefF, Machine learning 53 (1-2) (2003) 23-69.

[24] Y. Sun, J. Li, Iterative RELIEF for feature weighting, in: Proceedings of the 23rd international conference on Machine learning, ACM, 913-920, 2006.

[25] M. Srinivas, L. M. Patnaik, Adaptive probabilities of crossover and mutation in genetic algorithms, Systems, Man and Cybernetics, IEEE Transactions on 24 (4) (1994) 656-667.

[26] J. Zhang, H. S.-H. Chung, W.-L. Lo, Clustering-based adaptive crossover and mutation probabilities for genetic algorithms, Evolutionary Computation, IEEE Transactions on 11 (3) (2007) 326-335. 
[27] A. Koivunen, A. Kostinski, The feasibility of data whitening to improve performance of weather radar, Journal of Applied Meteorology 38 (6) (1999) 741-749.

[28] R. G. Brown, P. Y. Hwang, Introduction to random signals and applied Kalman filtering: with MATLAB exercises and solutions, Introduction to random signals and applied Kalman filtering: with MATLAB exercises and solutions, by Brown, Robert Grover.; Hwang, Patrick YC New York: Wiley, c1997. 1.

[29] A. Oustaloup, P. Melchior, P. Lanusse, O. Cois, F. Dancla, The CRONE toolbox for Matlab, in: Computer-Aided Control System Design, 2000. CACSD 2000. IEEE International Symposium on, IEEE, 190-195, 2000.

[30] C.-C. Chang, C.-J. Lin, LIBSVM: a library for support vector machines, ACM Transactions on Intelligent Systems and Technology (TIST) 2 (3) (2011) 27. 